\section{S26 RECURRENT RESPIRATORY TRACT INFECTIONS AND SPECIFIC ANTIBODY DEFICIENCY IN CHILDREN}

doi:10.1136/thx.2010.150912.26

M T C Lim, K Jeyarajah, H Pandya, M Browning, E Gaillard. University Hospitals of Leicester, Leicester, UK

Introduction Specific Antibody Deficiency ( $\mathrm{SAD}$ ) is associated with recurrent childhood lower respiratory tract infections (LRTI). SAD in children as a clinical entity is poorly defined, and has been described as a primary immunodeficiency of unknown origin. SAD can be identified by measuring antibodies to childhood vaccines, and a failure to respond to booster immunisations. The prevalence of $\mathrm{SAD}$ and its clinical significance in children is poorly understood.

Objectives The aim of this study is to determine the extent of SAD in children followed with recurrent respiratory tract infections at a tertiary paediatric respiratory centre.

Methods All patients with recurrent LRTI's attending our complex paediatric respiratory clinic over a 12-month period were studied. Results of specific antibody levels to pneumococcus and the response to booster vaccines were reviewed.

Results 102 children with recurrent respiratory infections were seen during this period (age range 10 months to 18 years, median age 4 years 10 months). Sixty-four children had specific IgG antibody levels to pneumococcus vaccine serotypes tested, of which $45(70 \%)$ had low antibody levels. Of these, 27 patients received either Prevenar (a polysaccharide conjugated vaccine containing seven serotypes) or Pneumovax II booster vaccines (a polysaccharide vaccine containing 23 pneumococcal serotypes), and were subsequently retested for their specific IgG antibody levels. Adequate levels of antibodies tested post-booster were mounted in only 13 patients (48\%)

Conclusions The prevalence of $\mathrm{SAD}$ in children with troublesome recurrent LRTI's is high and may be under-diagnosed. Pneumococcal vaccine booster response is variable in these children. The clinical significance of $\mathrm{SAD}$ and the response to booster immunisation warrants further study.

\section{S27 SHOULD ROUTINE SCREENING FOR VITAMIN D DEFICIENCY BE INCORPORATED INTO CURRENT GUIDELINES FOR TREATMENT OF PAEDIATRIC TB PATIENTS?}

doi:10.1136/thx.2010.150912.27

A Ting, M Jiki, C Bell, C Murray, Z Mughal, F Child. Royal Manchester Children's Hospital, Manchester, UK

Introduction and Objectives The association between low vitamin $\mathrm{D}$ levels and increased severity/susceptibility to TB infection due to suppression of innate immunity is well known. In the United Kingdom, low levels of background ultraviolet $B$ radiation limit the photoconversion of 7 dehydrocholestrol in skin to cholecalciferol (vitamin D3). In addition the majority of paediatric TB cases in the UK occur in non-Caucasian patients who by nature of their skin colour are less able to absorb the UV radiation. Current guidelines do not suggest routine checking of serum 25-dihydroxyvitamin D (25 $(\mathrm{OH}) \mathrm{D})$; a reliable measure of body stores of vitamin $\mathrm{D}$, in paediatric $\mathrm{TB}$ cases and providing Vitamin D supplementation as required. We hypothesised that all paediatric TB patients require screening for Vitamin $\mathrm{D}$ deficiency and that many would benefit from supplementation.

Methods This is a prospective cohort study. Between January 2009 and June 2010, all paediatric TB cases were prospectively recorded into a hospital database. Data collected include age, sex, serum concentrations of $25(\mathrm{OH}) \mathrm{D}$, calcium $(\mathrm{Ca})$, phosphate $(\mathrm{P})$, alkaline phosphatase (ALP) and parathyroid hormone (PTH).
Results 45 patients (18 males) were diagnosed with TB over the 18 month period. The mean age was 7.5 years (range 5 months to 17 years). The results for Vitamin D were not available in 5 patients (3 in progress, 2 not performed). Of the 40 patients with results available, $39(97.5 \%)$ were found to have low levels of $25(\mathrm{OH}) \mathrm{D}$ and were commenced on supplementation. Of these, $29 / 40(72.5 \%)$ had profound Vitamin D deficiency $(25(\mathrm{OH}) \mathrm{D}$ levels $<10 \mathrm{ng} / \mathrm{ml})$. Of these 29 patients, 15 had high PTH levels, 2 had low Ca (no data for one patient), 4 had low P levels and 5 had elevated ALP levels. One patient had radiologically confirmed rickets.

Conclusions Our study supports the incorporation of routine Vitamin D screening into the current guidelines for the management of $\mathrm{TB}$, especially as TB itself can worsen pre-existing vitamin $\mathrm{D}$ deficiency.

\section{REFERENCES}

1. Nnoaham KE, Clarke A. Low serum vitamin D levels and tuberculosis: a systematic review and meta-analysis. Int J Epidemiol 2008;37:113-19.

2. Martineau AR, Wilkinson RJ, Wilkinson KA, et al. A single dose of vitamin D enhances immunity to Mycobacteria. Am J Respir Crit Care Med 2007; 176:208-13.

\section{S28 CLINICAL PREDICTORS OF ADMISSION IN INFANTS WITH ACUTE BRONCHIOLITIS}

doi:10.1136/thx.2010.150912.28

M Marlais, J Evans, E Abrahamson. Chelsea and Westminster Hospital, London, UK

Introduction Bronchiolitis is a significant cause of acute morbidity in the first 12 months of life. A proportion of infants will be admitted to hospital and currently the decision to admit is based on clinical judgement alone. Research evidence identifying indicators for admission is scarce, there have been no studies assessing clinical predictors of admission in infants with bronchiolitis under the age of 12 months.

Aim To determine which clinical features in the emergency department are able to predict the need for hospital admission in infants with acute bronchiolitis.

Methods All infants presenting with acute bronchiolitis to one tertiary paediatric emergency department from April 2009 to March 2010 were included in this study. Inclusion criteria were a clinical diagnosis of bronchiolitis in an infant aged $<12$ months. The case notes for each infant were reviewed and data on 29 potential clinical predictors were recorded. An exhaustive list of potential clinical predictors was devised through literature review. Institutional approval was obtained to perform this study. Logistic regression analysis was performed to determine which clinical features could predict admission.

Results 449 infants presented with acute bronchiolitis during the study period (298 (66\%) male, mean age $23 \pm 14.5$ weeks). 163 (36\%) infants were admitted to hospital. The main clinical features predictive of admission (mean for admitted group vs discharged group respectively) included: younger age at presentation (mean 17.9 weeks vs 26.4 weeks, $p<0.001$ ), higher respiratory rate (mean 54.6 vs 46.9 , $\mathrm{p}<0.001$ ), higher heart rate (mean 162.8 vs $147.7, p<0.001$ ), lower oxygen saturation (mean $96.4 \%$ vs $98.4 \%, p<0.001$ ), lower duration of symptoms (mean 2.9 days vs 4.9 days, $\mathrm{p}<0.001$ ), lower weight at presentation (mean $6196 \mathrm{~g}$ vs $7897 \mathrm{~g}, \mathrm{p}<0.001$ ). The best single predictor of admission was oxygen requirement in the emergency department (OR 78.4 (95\% CI 18.8 to 326.9), $\mathrm{p}<0.001$ ).

Conclusion We have identified seven objective clinical parameters which can be used in the emergency department to predict which infants with bronchiolitis require admission to hospital. These data have been assimilated into a simple clinical risk score; further work is required to validate this scoring system and to assess its utility in supporting clinical decisions. 